\title{
Face Recognition Use Local Image Dataset and Correlation Technique
}

\author{
Dana Faiq Abd
}

Department of Information Technology, College of Science and Technology, University of Human Development, Sulaimani City, Iraq

\section{A B S T R A C T}

Face recognition is an extreme topic in security field which identifies humans through physiological or behavioral biometric characteristics. Face recognition can also identify the human almost in a precise detection; one of the primary problems in face recognition is the accurate recognition rate. Local datasets use for implementing this research rather than using public datasets. Midian filter uses to remove noise and identify errors, also obtains a good accuracy rate without modifying image quality. In addition, filter processing applies to modify and progress images and the discrete wavelet transforms algorithm uses as feature extraction. Many steps are applied in this approach such as image acquisition, converting images into gray scale, cropping the image, and then passing to the feature extraction. In order to get the final decision about the indicated face, some required steps are used in the comparison. The results show the accuracy of $91 \%$ of the recognition rate through the human face.

Index Terms: Face Recognition, Biometric Detection, Filter Processing, Finger Geometry, and Median Filter

\section{INTRODUCTION}

For many years biometric is one of the significant ways to use it to identify people. However, automatic biometric recognition appears and exists in recent years [1], [2]. Biological characteristics existing in every people however individual person is unique from these characteristics [3]. In addition, biological attributes get from the human can't be borrowed, forget, steal and replicate. Face recognition is one of the top methods in biometric identification because of its flexibility and user-friendly [4], [5]. There are some factors that directly impact faces recognition such as image illumination, quality of the image, type of camera, lens of the camera, and distance from the device to the face.

\begin{tabular}{|l|l|}
\hline Access this article online \\
\hline DOI: 10.21928/uhdjst.v5n2y2021.pp26-37 & $\begin{array}{l}\text { E-ISSN: 2521-4217 } \\
\text { P-ISSN: 2521-4209 }\end{array}$ \\
\hline
\end{tabular}

Copyright (c) $2021 \mathrm{Al}-\mathrm{Janabi}$, et al. This is an open access article distributed under the Creative Commons Attribution Non-Commercial No Derivatives License 4.0 (CC BY-NC-ND 4.0)
These elements make to achieve accurate authentication and verification. Furthermore, face recognition is the process to identified faces through computer systems and technologies [6], [7]. It is one of the most important security methods compares to traditional security [8]. In traditional security, users $\log$ in to the system using user names and passwords that can easily steal by hackers. Moreover, attackers fulfill different procedures to compromised and break traditional security [9], [10]. Besides, face recognition consists of some steps. The first step is a sensor; digital cameras are applied to capture images and collect data, and then transformed into digital format. In the next step, the signal processing algorithm is used to identifying, adjusting, improving and controlling data. Then, information should be stored inside a database, prepare for comparison and evaluation. Furthermore, processing and analyzing the images converting them to gray scale and cropped. Later, images separately send through the filtering process using a median filter. After that, an algorithm is applying to detect and compare retained templates. Finally, the automatic decision is performed based on the similarity and dissimilarity of collecting images. A special technique

Corresponding author's e-mail: Dana Faiq Abd, Assistant Lecturer, Department of Information Technology, College of Science and Technology, University of Human Development, Sulaimani City, Iraq. E-mail: dana.abd@uhd.edu.iq

Receiving: 01-05-2021

Accepting: 28-07-2021

Publishing: 05-08-2021 
is implemented such as Median filter and Filter processing to prevent photos from copying [11].

This research uses two effective techniques which are Median filter and Filter processing. Therefore, the main contribution of this research is to design a hybrid system by combining the discrete wavelet transforms (DWT), filter processing, and median filter these are leads to get a high accuracy recognition rate. The median filter is a method uses to erase, decrease noise, and identify faults on images or signals. In addition, it uses to enhance the quality of the pictures and to detecting duplicated noise. The median filter is a filtering method that focuses on smoothness, sharpness, and edge upgrading images or signals. On the other hand, Filter processing is a technique that is used to change and improve images. Besides, it can focus on any part of the images to remove or cropped unnecessary portions [12], [13]. Face recognition is the subject of many algorithms; the majority of them are solely concerned with the face recognition rate. The proposed method provides an effective face recognition algorithm that achieves a high recognition rate without affecting image quality. Implementing median filter and filter processing play a significant role to get a high accuracy recognition rate. Since the median filter is removing noise without affecting image quality.

Finally, the rest of the paper is organized as follows: Section 2 presents the literature review, section 3 discusses face recognition methods (proposed approach), and section 4 presents the results in the form of tables. Finally, section 5 discusses the conclusion.

\section{LITERATURE REVIEW}

There are numerous kinds of biometric innovations available such as face-acknowledgment, unique finger impression acknowledgment, finger geometry, hand geometry, iris acknowledgment, vein acknowledgment, voice, and mark. The face is the usually utilized biometric attribute for individual acknowledgment. Furthermore, the most prominent ways to deal with face acknowledgment depend on the state of facial characteristics. For example, eyes, eyebrows, nose, lips, jawline, and the connections of these qualities. The most well-established ID component is recognizing people by their facial highlights (or vectors), which may be traced back to our primate ancestors. The human capacity to perceive faces is likewise imperative to the security engineer due to the across-the-board dependence set on picture IDs.
Many papers and researches have been published which are associated with face recognition in this section. We will try to describe and discuss some of these related works and approaches:

Ahmed et al. (2019) implemented an approach to design efficient face recognition by blending many methods to generate efficient recognition results. This technique covers five stages. Image acquisition: for this stage, ORL picture Dataset has been applied to afford the pictures with diverse positions. Preprocessing: for this stage, several tasks have been done such as cleaning, resizing, and filtering. Then, singular value decomposition applied: for this stage, the actual picture is decomposed for three orthogonal matrices. Selecting the most effective rank: For this step, the measurement of dispersion is used to present the best efficient matrix rank is achieved. In addition, the DW'T applied: in this stage, the diagonal matrix produces significant characteristics by applied the DWT. Later, measures of dispersion are used to imply the division of data. According to the applied ranks, there is an acceptable reasonable rank that is important to reach via the implemented procedure. Interquartile range, mean absolute deviation, range, variance, and standard deviation are used to pick the correct rank. Rank 24, 12, and 6 acquired an outstanding 100\% identification rate with data decrease up to $2: 1,4: 1$, and $8: 1$ correspondingly. The regular PC and MATLAB simulator tool version 2015 used to complete the task [14]. Belhumeur et al. (1997), focus on developing a face recognition algorithm. Even under the substantial change in lighting and facial appearances, their projection method focuses on developing a face recognition algorithm for facial expression and light direction which is obtuse to great deviation. Consider each pixel in an image as a coordinate in a high-dimensional space when using a pattern categorization method. There are some gained advantages of the fact that images of a particular face, under fluctuating light but fixed position, reside in a 3D linear subspace of the high-dimensional image space. Supposing the face is a Lambertian (diffusely reflecting) surface with no shadows. Images will diverge from this linear subspace since faces are not fully Lambertian surfaces and create selfshadowing. They linearly project the image into a subspace which is based on Fisher's linear discriminant. Produces well-separated classes in a low-dimensional subspace [15]. Chen et al. (2014) Face recognition has a large dictionary and a unique value. The intra-class variation dictionary is used in the study in a subsampling state to represent the difference between samples in both the learning and examination phases [16]. Dehghan et al. (2015) used a portion of the training data for data modeling and used a knowledge 
score to define the result, which are features of confidence representation [17]. Zheng et al. (2016) developing a comprehensive divided representation dictionary for facial recognition that can effectively overcome occlusion issues [18]. Gao et al. (2017) offered a new strategy by generating a low range of the data representation and each fault image generated by the occlusion in real-time [19]. Wu and Ding (2018) resolved the issue of face recognition, a hierarchical model based on Adaptive Sparse and LowRank gradients was used to solve the problem of face recognition [20]. Jing et al. (2019) apply a different method to find the discriminant tensor demonstration to properly apply the photo taxonomy problem [21]. Wadkar et al. (2012) developed a facial recognition technique based on DWT. To understand a certain range wavelet, decompositions are used in the procedure in order to improve performance. Furthermore, as a part of the approach algorithm, Haar, $9 / 7$ wavelet filters used in the research. The work engages a variety of distance measures, including Euclidean, L1, and others. The Euclidean distance is obtained popularity [22]. Dandpat et al. (2013) used both conventional principal component analysis (PCA) and two-dimensional PCA (2DPCA) to increase face recognition performance. Their method shows that eigenvectors with limited nonzero eigenvalues are related. Different weights should be given to non-trivial fundamental parts for each category. Finally, the $\mathrm{k}$-nearest-distance method is applied to determine facial recognition [23]. The proposed method is based on the MATLAB simulation tool and applied DWT. Furthermore, low pass band and high pass band applied on both rows and columns, respectively, using a median filter. Moreover, remarkable biometric distinguishing obtains by applying multiple biometric techniques like face, voice, or Iris, face. These highlights have their particular favorable circumstances and burdens with respect to the applications and necessities [24]. One acknowledgment framework that functions admirably for a specific prerequisite may not be appropriate to other people. Among these component acknowledgment frameworks, face acknowledgment likewise finds significance from the previous year's spreading over various fields. Face acknowledgment is vital on the grounds that it finds a few down-to-earth applications. Also, it is a key human conduct for communicating feelings [25]. The utilization of face as a component for acknowledgment framework additionally has preferences in law implementation. As it doesn't require dynamic support from the individual as enormous information test assortment is reachable in a helpful manner [26]. In the end, median filter has some advantages and disadvantages. The advantages median filter is one of the most important filters because it works well with noise types including "Gaussian," "random," and "salt and pepper." Noise pixels are distinguished from the median. This type of noise problem can be eliminated by using this approach median filter. When use low pass filter in median filter only the noise reduces contrary all other type of filter reduces the image quality [27]. When the noise amplitude probability distribution contains big tails and periodic patterns, median filters are beneficial for decreasing random noise. In addition, when apply median filter there is no need to create a new pixel value and it is simple to put into practice. The downsides of such filters are that they tend to break up image edges and produce misleading noise edges when signal-to-noise ratios are low, and they cannot suppress medium-tailed (Gaussian) noise distributions. Finally, the median filter causes some delay in processing time [28], [29].

\section{THE PROPOSED APPROACH}

\subsection{Local Image Dataset}

For data collection and image acquisition, ten volunteers are prepared in a suitable area with a natural environment without external lighting factors. Besides, all volunteers are between 20 and 30 years old, take pictures using an ordinary camera for each volunteer. Four images of head captured that include all front hair, forehead, eyebrow, eyelash, eye, ears, nose, cheek, mouth, lip, and chin.

HUAWEI Y6II mobile phone camera, with resolution 4160 $\times 3120$ and 13 mega pixels' technology used in the procedure with a distance of $2-\mathrm{m}$ in between mobile phone camera and volunteer.

\subsection{DWT}

As shown in Fig. 1, the DWT distributes an image into four groups such as approximation band, vertical band, horizontal band, and diagonal band. The maximum number of levels equal to $\mathrm{N} \times \mathrm{N}$ image size. Both the row and columns of images traversing across the low path and high pass filter to create low and high-frequency approximation and vertical bands consequently. Besides, the column crossing through low pass and high pass filter with results of row high pass filter to make horizontal and diagonal bands. The LL band includes important information about an original image. The $\mathrm{LH}, \mathrm{HL}$, and $\mathrm{HH}$ bands have vertical, horizontal, and diagonal information of an original image. The original image recreated by deliberately only the LL band image and eliminating other unimportant information from other bands [30], [31]. 
A dataset with forty images obtained (four images for each volunteer), for preparation of processing on the face only, as shown in Fig. 2.

\subsection{Implemented Approach}

Image acquisition, converting images from color images to gray scale images, cropping image by a rectangle with $1000 \times 1000$ pixels for face images as demonstrate in Fig. 3 . Images composed of eyes, nose, mouth, and cheeks are ongoing steps implemented in the proposed approach for face recognition. Each rectangle image separately passed via filtering process using median filter (which is a non-linear digital filtering method used in a stage of preprocessing to remove noise from an image). A median filter has very robust efficiency which concurrently can decrease noise and retain edges. The median filter makes evaluation and computation based on adjacent pixels which select the middle value of a dataset listed from smallest to the largest. It examines the entire original image from top to bottom, left to the right, and makes a particular image that is based on median values.

Fig. 4 is an example of a median filter which is by default it collects the adjacency pixels around the target pixel, and

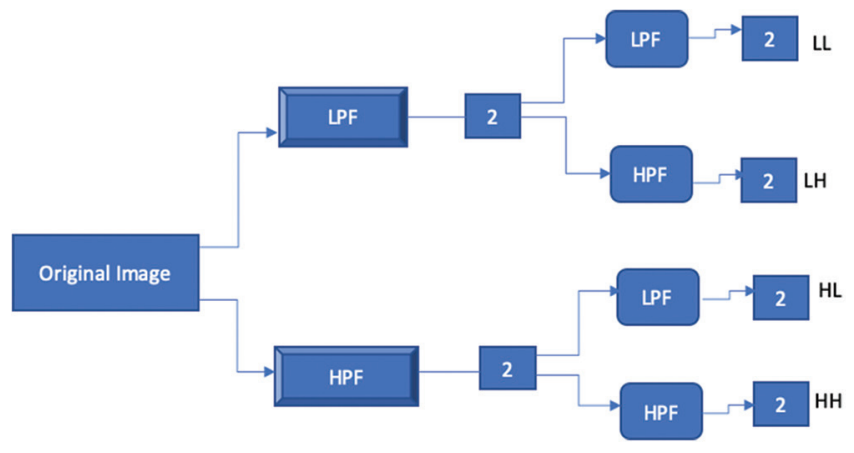

Fig. 1. Decomposition of discrete wavelet transforms. categorizing them from small value to high value, and picks the median value from the arranged list. As demonstrated in Fig. 4 from the output window, there are different values and from sorter out the values are sorting from smallest to the highest value, and then from output pixel, the median value is selected.

According to the research procedure of data collection, there are 40 images collected for the face. There are 40 volunteers, for each volunteer four different images uses. The value of correlation between each pair of images recorded to each volunteer. Finally, the correlation value calculated for each face image as shown in Figs. 4 and 5. Because for each volunteer four images captured then perform processing and applying the medium filter for each image. The correlation value between images is calculated. As discussed in (3.4 Discussion).

Fig. 4 is illustrating an image face for a volunteer the first one is the image face before filtering and the second one is the image face after the filtering process.

\section{DISCUSSION}

Although comparisons have been done all images, respectively, the variance between both images is small or big depending on the details of the image, in this situation; the median filter plays an important role in the identification process. When the result value (RV) obtained for each pair of images, consequently added to a table to show the degree of similarity among all pictures taken for each volunteer as shown in Table 1.

- $\quad \mathrm{N}^{\text {th }}$ : Number of volunteers (we have 10 volunteers).

- $\quad 1^{\text {st }}$ pic: Volunteer first picture.

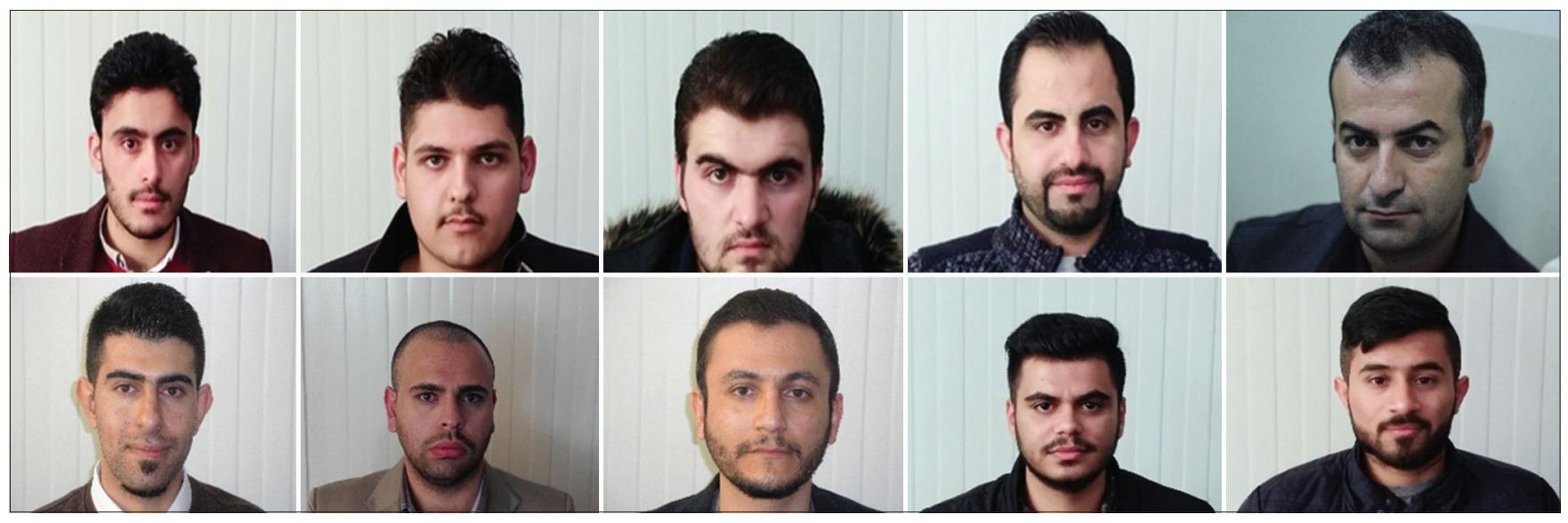

Fig. 2. A face image dataset. 


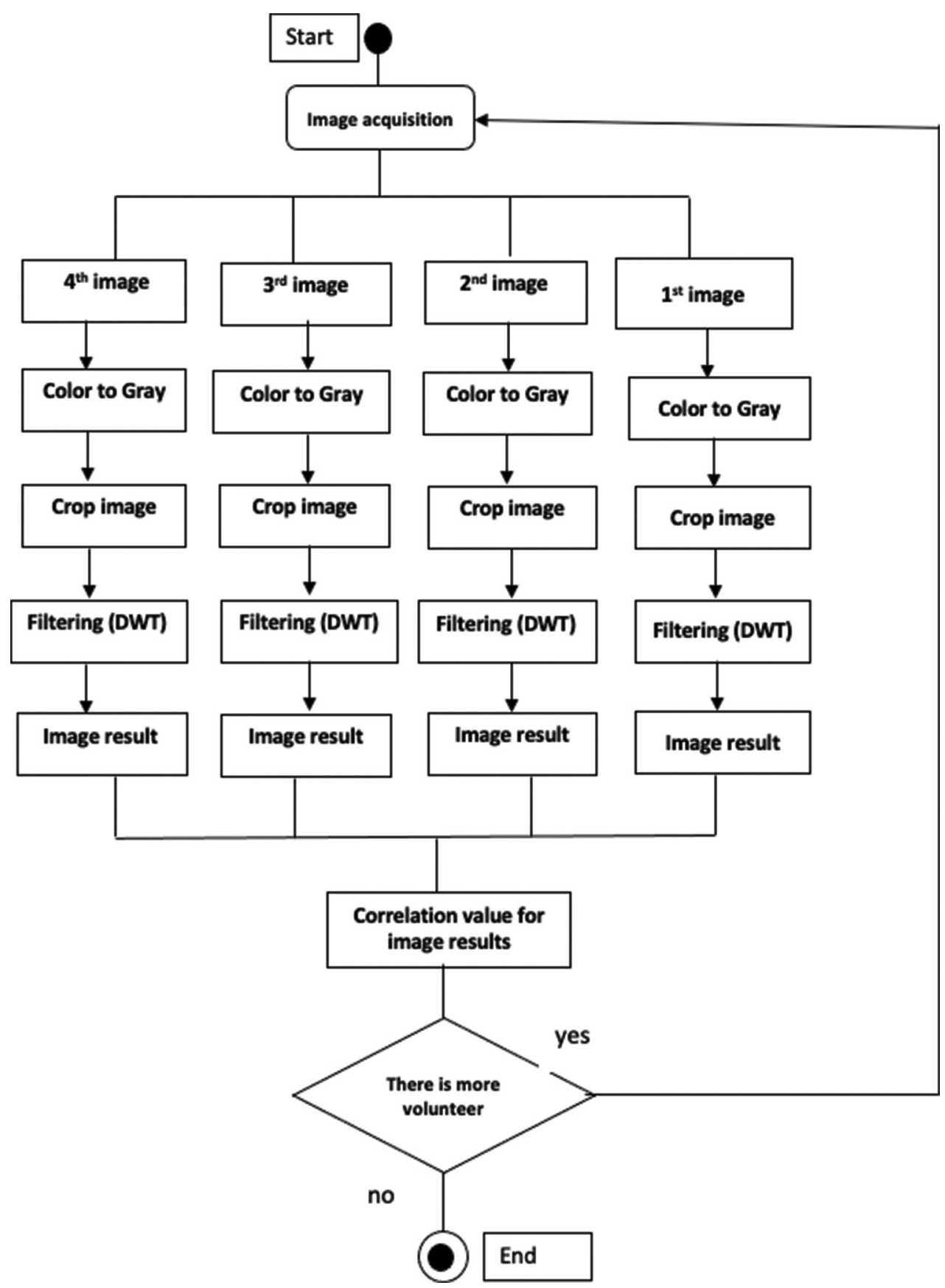

Fig. 3. Main steps of methodology.

TABLE 1: The result values of correlation

\begin{tabular}{|c|c|c|c|c|c|}
\hline & & $1^{\text {st }}$ pic & $2^{\text {nd }}$ pic & $3^{\text {rd }}$ pic & $4^{\text {th }}$ pic \\
\hline \multirow[t]{3}{*}{ Number of volunteer Face Images } & $1^{\text {st }}$ pic & Result value 1 & Result value 2 & Result value 3 & Result value 4 \\
\hline & $2^{\text {nd }}$ pic & Result value 1 & Result value 2 & Result value 3 & Result value 4 \\
\hline & $3^{\text {rd }}$ pic & Result value 1 & Result value 2 & Result value 3 & Result value 4 \\
\hline
\end{tabular}


- $\quad 2^{\text {nd }}$ pic: Volunteer second picture.

- $\quad 3^{\text {rd }}$ pic: Volunteer third picture.

- $4^{\text {th }}$ pic: Volunteer fourth picture.

- RV: Correlation value between each image in a row with its corresponding image in a column.

To be more familiar with understanding the results, there is a chart graph for each table of result ..... to show similarity between images as shown in Fig. 7.

- Blue column represents Correlation RV between the volunteer First picture with other pictures in the first column of the table.

- Brown column represents Correlation RV between volunteer Second picture with other pictures in Second column of the table.

- Gray column represents Correlation RV between volunteer Third picture with other pictures in the third column of the table.

- Yellow column represents Correlation RV between volunteer Fourth picture with other pictures in Second column of the table.

- Each aggregated 4 column in the $\mathrm{X}$-axis represents a unique row of the table.

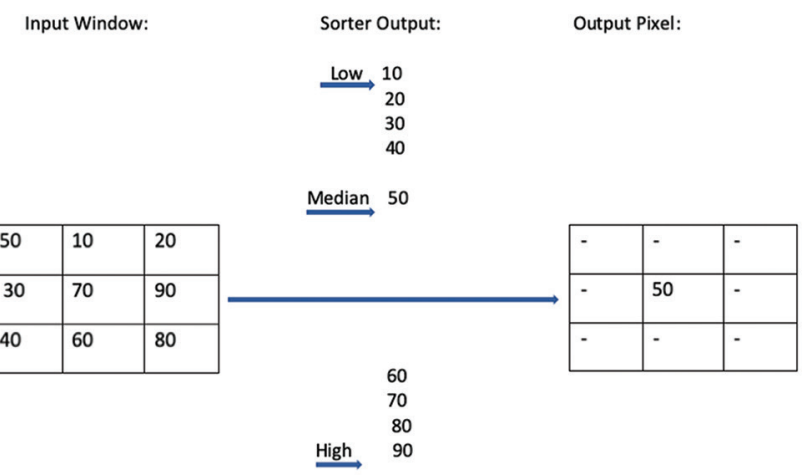

Fig. 4. The median filtering algorithm.
- The degrees located at Y-axis represent the value result.

\section{RESULTS}

According to the implementation of the proposed system, many results obtained according to the tested samples. These results are the correlation between different images, similarities, and differences to achieve the identification process. Considering the range of (1.0-0.5) enumerate as a positive correlation, which indicated the best identification result between different tested images. Then considering the range from 0.5 to the minimum value, this means a negative correlation that has no relation between the tested images. According to the obtained results from ten samples of face images, the result is $91 \%$ for all correlation RVs for face processed with a median filter. Good results of identification and differentiation in biometry process obtained.

The blue column represents the correlation RV between the volunteer's first pictures with other pictures in the first column of the table. The Brown column represents the correlation RV between the volunteer second pictures with other pictures in the second column of the table. The Gray column represents the correlation $\mathrm{RV}$ between the volunteer third picture with other pictures in the third column of the table. The yellow column represents the correlation RV between the volunteer fourth pictures with other pictures in the second column of the table. The black line represents a total average correlation. The degrees located at Y-axis represent the value result.

As shown from Table 2 and Fig. 8 below, Correlation average for $1^{\text {st }}$ volunteer Face images shows RV of 0.9 for each image with its corresponding person images, which is

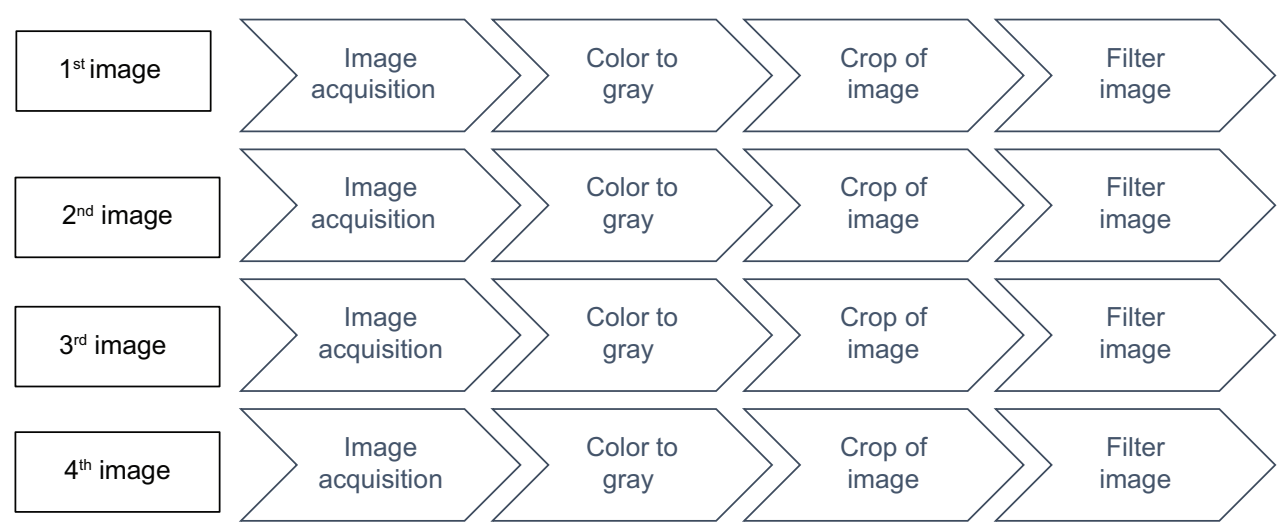

Fig. 5. Implemented approach. 
a positive result according to the range mentioned above. Blue column represents correlation RV between volunteer first pictures with other pictures in first column of the table. Brown column represents correlation RV between volunteer second picture with other pictures in second column of the table. Gray column represents correlation RV between volunteer third picture with other pictures in third column of the table. Yellow column represents correlation RV between volunteer fourth picture with other pictures in second column of the table. The black line represents total of average correlation. The degrees located at Y-axis represent value result.

As shown from Table 3 and Fig. 9 below, Correlation average for $2^{\text {nd }}$ volunteer Face images shows RV of 0.9 for each image with its corresponding person images, which is a positive result according to the range mentioned above. Blue column represents correlation RV between volunteer first picture with other pictures in first column of the table. Brown column represents correlation RV between volunteer second picture with other pictures in second column of the table. Gray column represents correlation RV between volunteer third picture with other pictures in third column of the table. Yellow column represents correlation $\mathrm{RV}$ between volunteer fourth picture with other pictures in second column of the table. The black line represents total of average correlation. The degrees located at Y-axis represent value result.

As shown from Table 4 and Fig. 10 below, Correlation average for $3^{\text {rd }}$ volunteer Face images shows RV of 1.0 for each image with its corresponding person images, which is a positive result according to the range mentioned above. Blue column represents correlation RV between volunteer

\section{TABLE 2: Correlation value for $1^{\text {st }}$ volunteer FACE} images

\begin{tabular}{lccccc}
\hline & & $\mathbf{1}^{\text {st }} \mathbf{p i c}$ & $\mathbf{2}^{\text {nd }} \mathbf{p i c}$ & $\mathbf{3}^{\text {rd }} \mathbf{p i c}$ & $\mathbf{4}^{\text {th }} \mathbf{p i c}$ \\
\hline $1^{\text {st }}$ Volunteer & $1^{\text {st }}$ pic & 1 & 0.8765 & 0.8574 & 0.8961 \\
FACE image & $2^{\text {nd }}$ pic & 0.8765 & 1 & 0.7032 & 0.9882 \\
& $3^{\text {rd }}$ pic & 0.8574 & 0.7032 & 1 & 0.7348 \\
& $4^{\text {th }}$ pic & 0.8961 & 0.9882 & 0.7348 & 1 \\
\hline
\end{tabular}

TABLE 3: Correlation value for $2^{\text {nd }}$ volunteer FACE images

\begin{tabular}{lccccc}
\hline & & $\mathbf{1}^{\text {st }} \mathbf{p i c}$ & $\mathbf{2}^{\text {nd }} \mathbf{p i c}$ & $\mathbf{3}^{\text {rd }} \mathbf{p i c}$ & $\mathbf{4}^{\text {th }} \mathbf{p i c}$ \\
\hline $2^{\text {nd }}$ Volunteer & $1^{\text {st }}$ pic & 1 & 0.8862 & 0.8761 & 0.8633 \\
FACE image & $2^{\text {nd }}$ pic & 0.8862 & 1 & 0.9452 & 0.9359 \\
& $3^{\text {rd }}$ pic & 0.8761 & 0.9452 & 1 & 0.9911 \\
& $4^{\text {th }}$ pic & 0.8633 & 0.9359 & 0.9911 & 1 \\
\hline
\end{tabular}

first picture with other pictures in first column of the table. Brown column represents correlation RV between
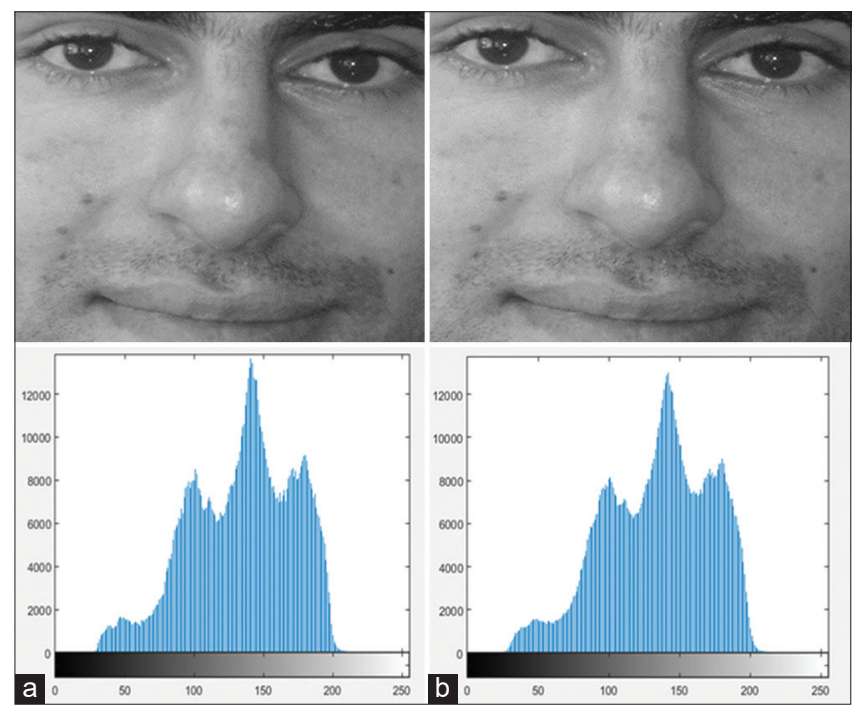

Fig. 6. ( $a$ and b) Faces image before and after filtering.

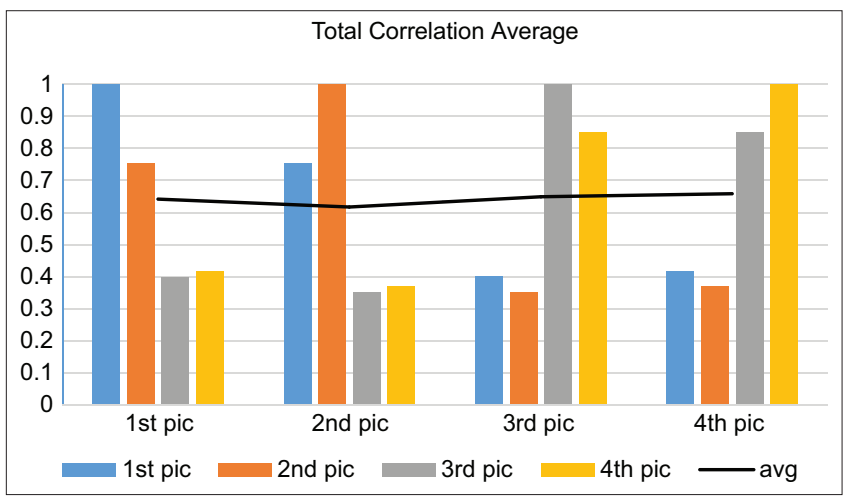

Fig. 7. The result average value of the correlation.

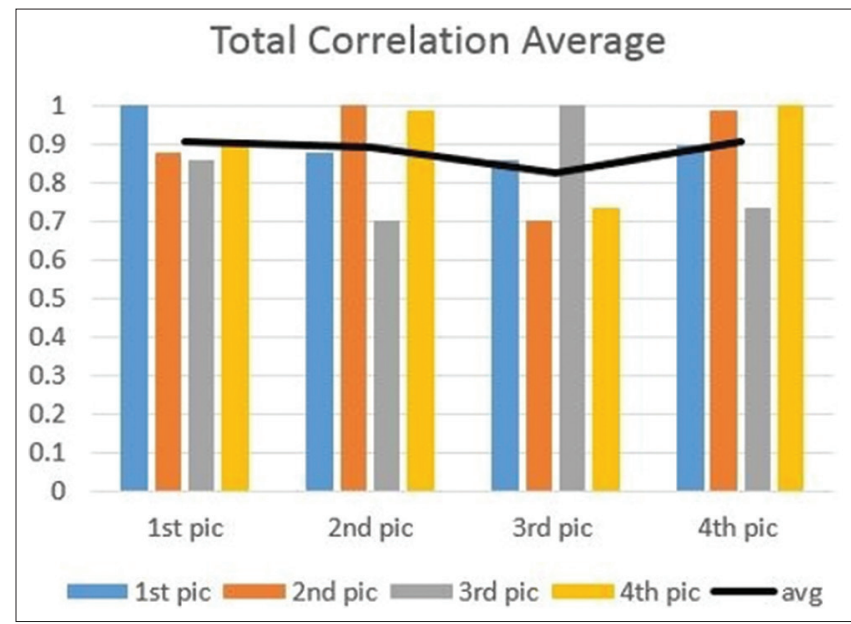

Fig. 8. Average correlation value for $1^{\text {st }}$ volunteer FACE images. 


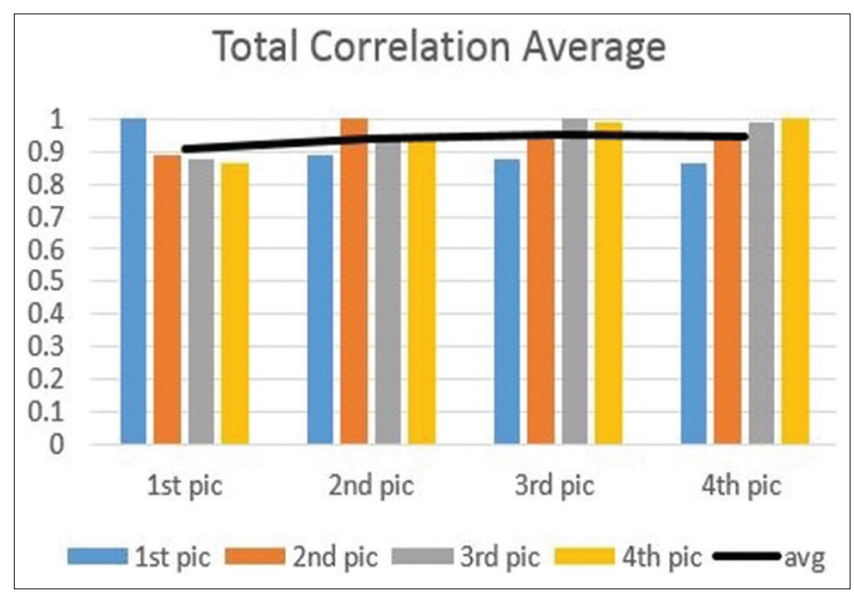

Fig. 9. Average Correlation value for $2^{\text {nd }}$ volunteer FACE images.

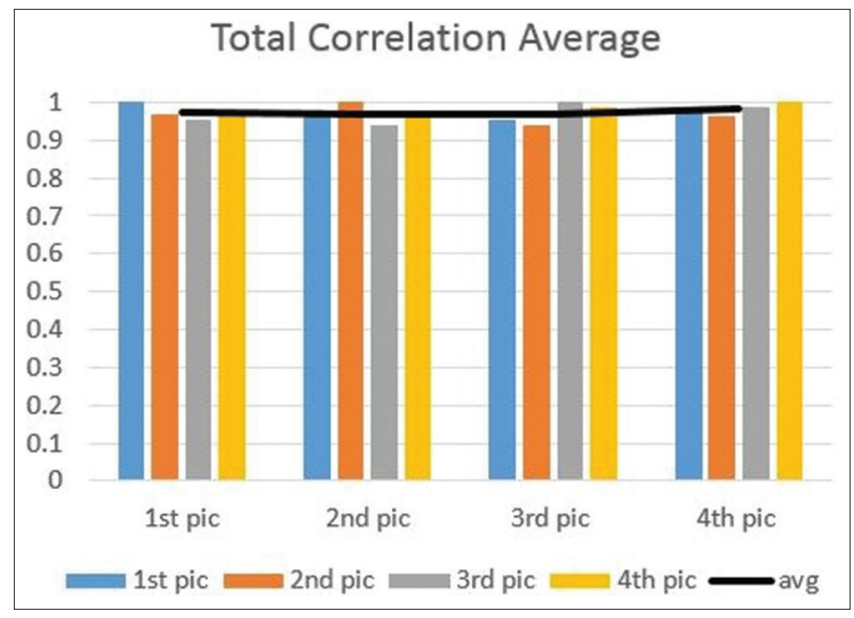

Fig. 10. Average correlation value for $3^{\text {rd }}$ volunteer FACE images.

volunteer second picture with other pictures in second column of the table. Gray column represents correlation RV between volunteer third picture with other pictures in third column of the table. Yellow column represents correlation RV between volunteer fourth picture with other pictures in second column of the table. The black line represents total of average correlation. The degrees located at Y-axis represent value result.

As shown from Table 5 and Fig. 11 below, Correlation average for $4^{\text {th }}$ volunteer Face images shows RV of 0.9 for each image with its corresponding person images, which is a positive result according to the range mentioned above. Blue column represents correlation RV between volunteer first picture with other pictures in first column of the table. Brown column represents correlation RV between volunteer second picture with other pictures in second column of the table. Gray column represents correlation RV between volunteer

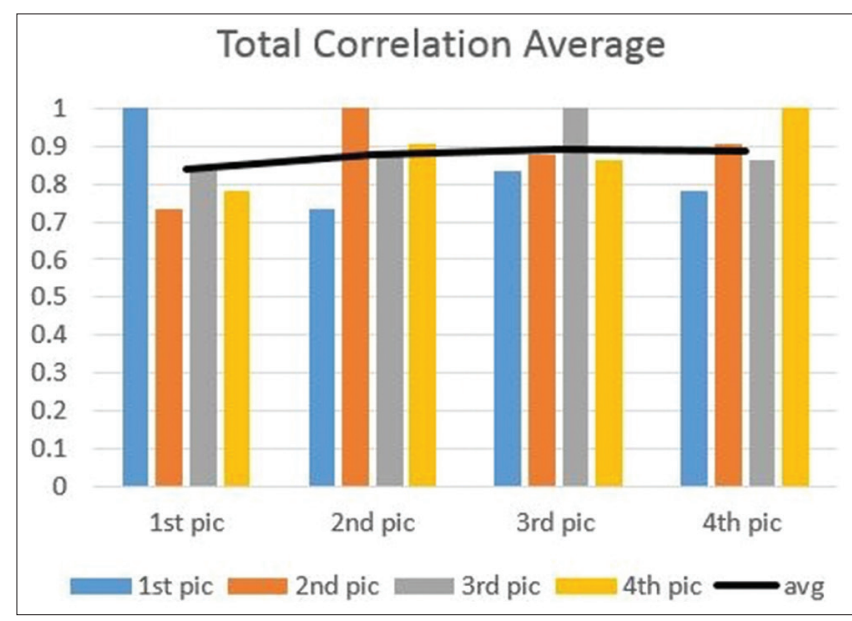

Fig. 11. Average correlation value for $4^{\text {th }}$ volunteer FACE images.

\begin{tabular}{|c|c|c|c|c|c|}
\hline & & $1^{\text {st }}$ pic & $2^{\text {nd }}$ pic & $3^{\text {rd }}$ pic & $4^{\text {th }}$ pic \\
\hline $3^{\text {rd }}$ Volunteer & $1^{\text {st }}$ pic & 1 & 0.9668 & 0.9548 & 0.9763 \\
\hline \multirow[t]{3}{*}{ FACE image } & $2^{\text {nd }}$ pic & 0.9668 & 1 & 0.938 & 0.9631 \\
\hline & $3^{\text {rd }}$ pic & 0.9548 & 0.938 & 1 & 0.9892 \\
\hline & $4^{\text {th }}$ pic & 0.9763 & 0.9631 & 0.9892 & 1 \\
\hline
\end{tabular}

TABLE 5: Correlation value for $4^{\text {th }}$ volunteer FACE images

\begin{tabular}{llcccc}
\hline & & $\mathbf{1}^{\text {st }} \mathbf{p i c}$ & $\mathbf{2}^{\text {nd }} \mathbf{p i c}$ & $\mathbf{3}^{\text {rd }} \mathbf{p i c}$ & $\mathbf{4}^{\text {th }} \mathbf{p i c}$ \\
\hline $4^{\text {th }}$ Volunteer & $1^{\text {st }}$ pic & 1 & 0.7339 & 0.8339 & 0.7822 \\
FACE image & $2^{\text {nd }}$ pic & 0.7339 & 1 & 0.8757 & 0.9069 \\
& $3^{\text {rd }}$ pic & 0.8339 & 0.8757 & 1 & 0.8611 \\
& $4^{\text {th }}$ pic & 0.7822 & 0.9069 & 0.8611 & 1 \\
\hline
\end{tabular}

third picture with other pictures in third column of the table. Yellow column represents correlation RV between volunteer fourth picture with other pictures in second column of the table. The black line represents total of average correlation. The degrees located at Y-axis represent value result.

As shown from Table 6 and Fig. 12 below, Correlation average for $5^{\text {th }}$ volunteer Face images shows RV of 0.9 for each image with its corresponding person images, which is a positive result according to the range mentioned above. Blue column represents correlation RV between volunteer first picture with other pictures in first column of the table. Brown column represents correlation RV between volunteer second picture with other pictures in second column of the table. Gray column represents correlation RV between volunteer third picture with other pictures in third column of the table. Yellow column represents correlation RV between volunteer 
fourth picture with other pictures in second column of the table. The black line represents total of average correlation. The degrees located at Y-axis represent value result.

As shown from Table 7 and Fig. 13 below, Correlation average for $6^{\text {th }}$ volunteer Face images shows RV of 1.0 for each image with its corresponding person images, which is a positive result according to the range mentioned above. Blue column represents correlation RV between volunteer first picture with other pictures in first column of the table. Brown column represents correlation RV between volunteer second picture with other pictures in second column of the table. Gray column represents correlation RV between volunteer third picture with other pictures in third column of the table. Yellow column represents correlation RV between volunteer fourth picture with other pictures

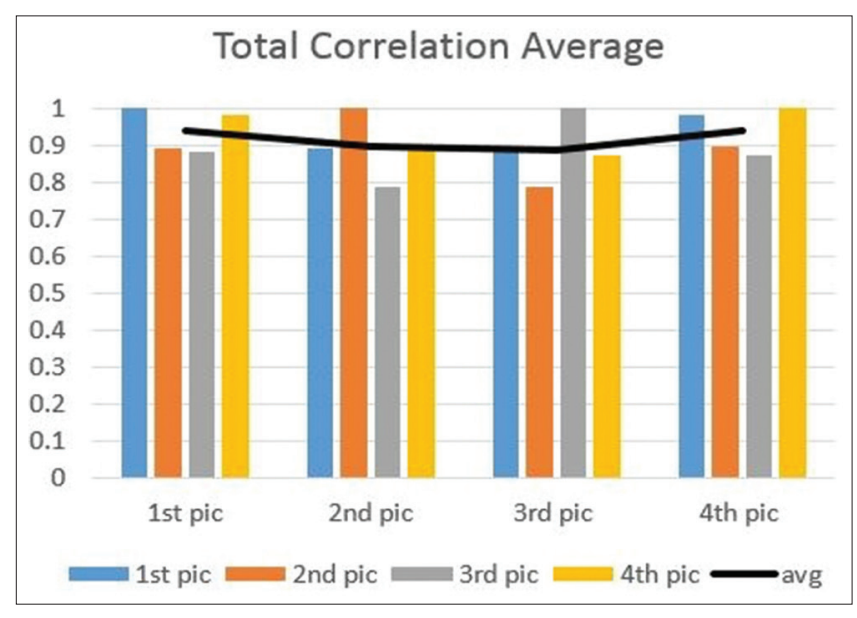

Fig. 12. Average correlation value for $5^{\text {th }}$ volunteer FACE images.

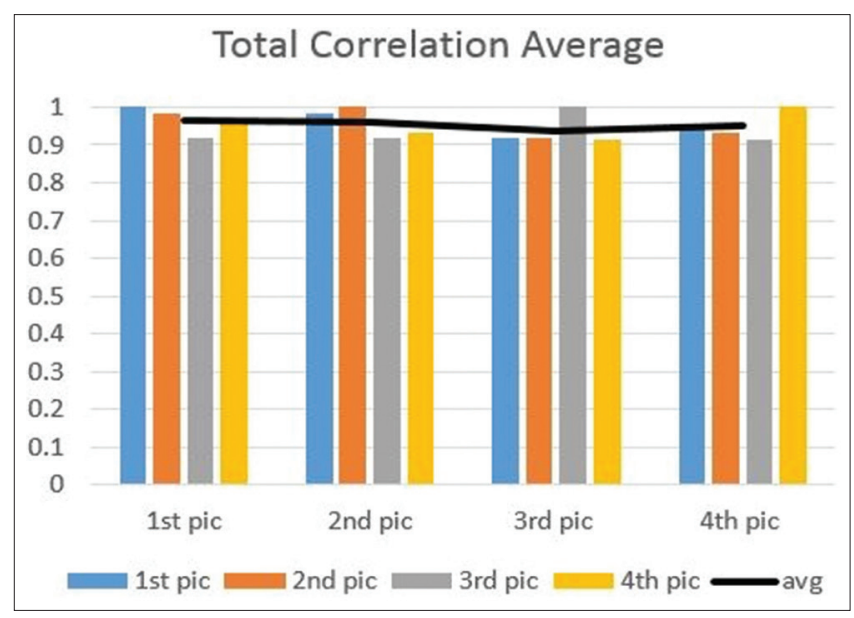

Fig. 13. Average correlation value for $6^{\text {th }}$ volunteer FACE images. in second column of the table. The black line represents total of average correlation. The degrees located at Y-axis represent value result.

As shown from Table 8 and Fig. 14 below, Correlation average for $7^{\text {th }}$ volunteer Face images shows RV of 0.8 for each image with its corresponding person images, which is a positive result according to the range mentioned above. Blue column represents correlation RV between volunteer first picture with other pictures in first column of the table. Brown column represents correlation RV between volunteer second picture with other pictures in second column of the table. Gray column represents correlation RV between volunteer third picture with other pictures in third column of the table. Yellow column represents correlation RV between volunteer fourth picture with other pictures in second column of the table. The black line represents total of average correlation. The degrees located at Y-axis represent value result.

As shown from Table 9 and Fig. 15 below, Correlation average for $8^{\text {th }}$ volunteer Face images shows RV of 0.9 for each image with its corresponding person images, which is a positive result according to the range mentioned above.

TABLE 6: Correlation value for $5^{\text {th }}$ volunteer FACE images

\begin{tabular}{lccccc}
\hline & & $\mathbf{1}^{\text {st }} \mathbf{p i c}$ & $\mathbf{2}^{\text {nd }} \mathbf{p i c}$ & $\mathbf{3}^{\text {rd }} \mathbf{p i c}$ & $\mathbf{4}^{\text {th }} \mathbf{p i c}$ \\
\hline $5^{\text {th }}$ Volunteer & $1^{\text {st }}$ pic & 1 & 0.8907 & 0.8833 & 0.9845 \\
FACE image & $2^{\text {nd }}$ pic & 0.8907 & 1 & 0.789 & 0.8994 \\
& $3^{\text {rd }}$ pic & 0.8833 & 0.789 & 1 & 0.8723 \\
& $4^{\text {th }}$ pic & 0.9845 & 0.8994 & 0.8723 & 1 \\
\hline
\end{tabular}

TABLE 7: Correlation value for $\mathbf{6}^{\text {th }}$ volunteer FACE images

\begin{tabular}{lccccc}
\hline & & $\mathbf{1}^{\text {st }} \mathbf{p i c}$ & $\mathbf{2}^{\text {nd }} \mathbf{p i c}$ & $\mathbf{3}^{\text {rd }} \mathbf{p i c}$ & $\mathbf{4}^{\text {th }} \mathbf{p i c}$ \\
\hline $6^{\text {th }}$ Volunteer & $1^{\text {st }}$ pic & 1 & 0.9811 & 0.918 & 0.9543 \\
FACE image & $2^{\text {nd }}$ pic & 0.9811 & 1 & 0.9197 & 0.9309 \\
& $3^{\text {rd }}$ pic & 0.918 & 0.9197 & 1 & 0.9119 \\
& $4^{\text {th }}$ pic & 0.9543 & 0.9309 & 0.9119 & 1 \\
\hline
\end{tabular}

TABLE 8: Correlation value for $7^{\text {th }}$ volunteer FACE images

\begin{tabular}{lccccc}
\hline & & $\mathbf{1}^{\text {st }} \mathbf{p i c}$ & $\mathbf{2}^{\text {nd }} \mathbf{p i c}$ & $\mathbf{3}^{\text {rd }} \mathbf{p i c}$ & $\mathbf{4}^{\text {th }} \mathbf{p i c}$ \\
\hline $7^{\text {th }}$ Volunteer & $1^{\text {st }}$ pic & 1 & 0.867 & 0.9224 & 0.6389 \\
FACE image & $2^{\text {nd }}$ pic & 0.9224 & 1 & 0.7905 & 0.5321 \\
& $3^{\text {rd }}$ pic & 0.9224 & 0.7905 & 1 & 0.7209 \\
& $4^{\text {th }}$ pic & 0.6389 & 0.5321 & 0.7209 & 1 \\
\hline
\end{tabular}




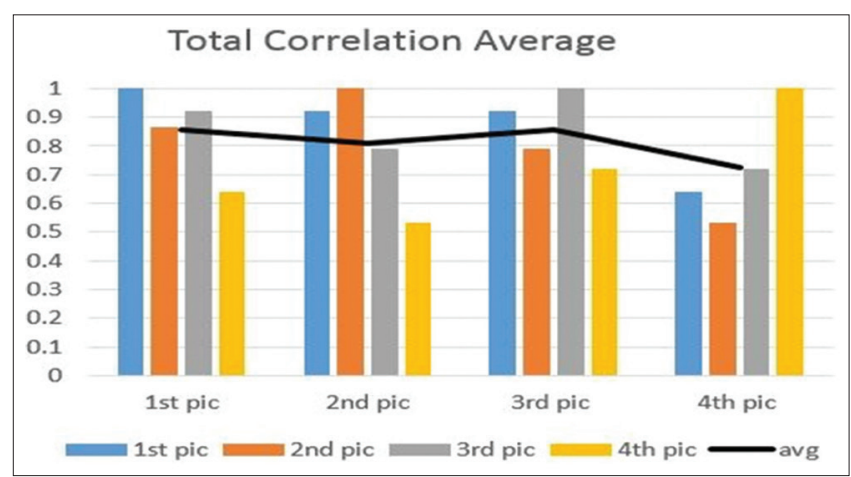

Fig. 14. Average correlation value for $7^{\text {th }}$ volunteer FACE images.

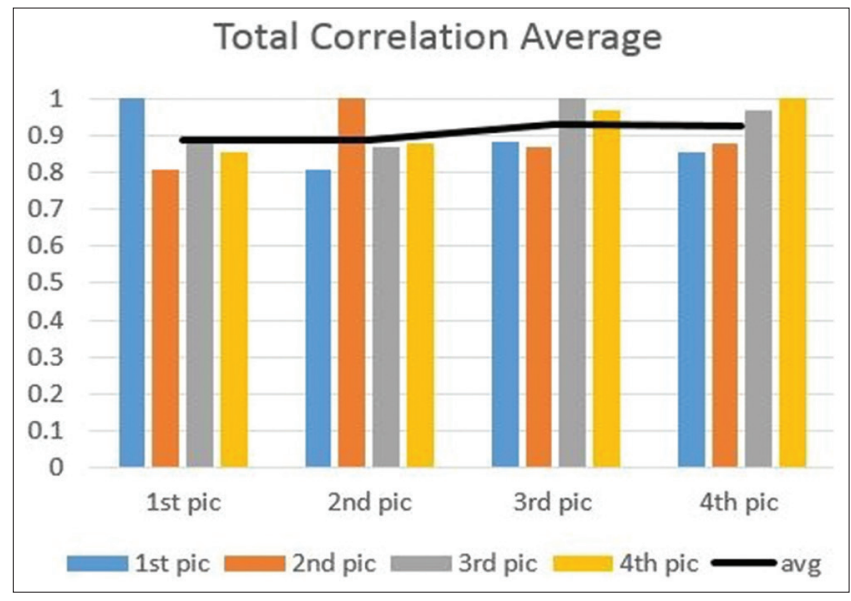

Fig. 15. Average correlation value for $8^{\text {th }}$ volunteer FACE images.

Blue column represents correlation RV between volunteer first picture with other pictures in first column of the table. Brown column represents correlation RV between volunteer second picture with other pictures in second column of the table. Gray column represents correlation RV between volunteer third picture with other pictures in third column of the table. Yellow column represents correlation RV between volunteer fourth picture with other pictures in second column of the table. The black line represents total of average correlation. The degrees located at Y-axis represent value result.

As shown from Table 10 and Fig. 16 below, Correlation average for $9^{\text {th }}$ volunteer Face images shows RV of 0.9 for each image with its corresponding person images, which is a positive result according to the range mentioned above. Blue column represents correlation RV between volunteer first picture with other pictures in first column of the table. Brown column represents correlation RV between volunteer second picture with other pictures in second column of the table. Gray column represents correlation RV

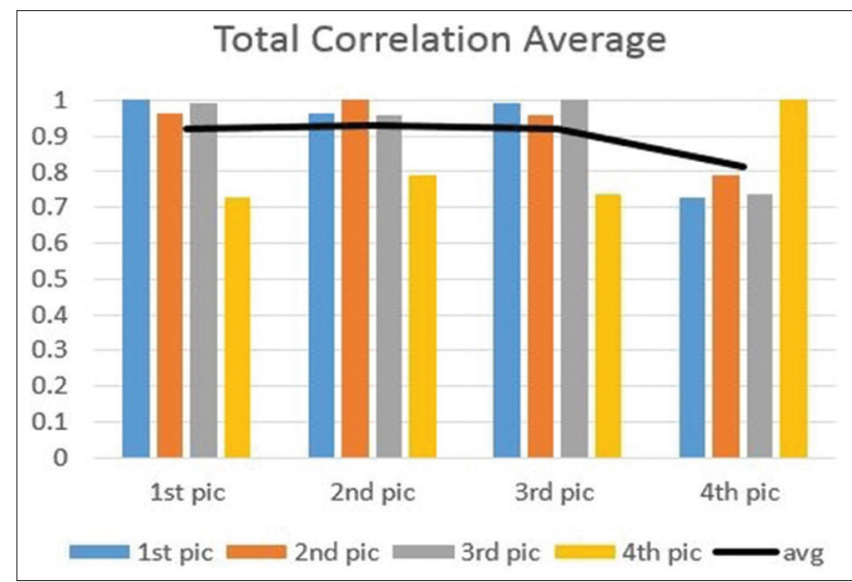

Fig. 16. Average correlation value for $9^{\text {th }}$ volunteer FACE images.

\begin{tabular}{|c|c|c|c|c|c|}
\hline & & $1^{\text {st }}$ pic & $2^{\text {nd }}$ pic & $3^{\text {rd }}$ pic & $4^{\text {th }}$ pic \\
\hline $8^{\text {th }}$ Volunteer & $1^{\text {st }}$ pic & 1 & 0.8087 & 0.8857 & 0.8572 \\
\hline \multirow[t]{3}{*}{ FACE image } & $2^{\text {nd }}$ pic & 0.8087 & 1 & 0.869 & 0.8778 \\
\hline & $3^{\text {rd }}$ pic & 0.8857 & 0.869 & 1 & 0.9703 \\
\hline & $4^{\text {th }}$ pic & 0.8572 & 0.8778 & 0.9703 & 1 \\
\hline
\end{tabular}

TABLE 10: Correlation value for $9^{\text {th }}$ volunteer FACE images

\begin{tabular}{lccccc}
\hline & & $\mathbf{1}^{\text {st }} \mathbf{p i c}$ & $\mathbf{2}^{\text {nd }} \mathbf{p i c}$ & $\mathbf{3}^{\text {rd }} \mathbf{p i c}$ & $\mathbf{4}^{\text {th }} \mathbf{p i c}$ \\
\hline $9^{\text {th }}$ Volunteer & $1^{\text {st }}$ pic & 1 & 0.9634 & 0.991 & 0.7296 \\
FACE image & $2^{\text {nd }}$ pic & 0.9634 & 1 & 0.9576 & 0.7892 \\
& $3^{\text {rd }}$ pic & 0.991 & 0.9576 & 1 & 0.7373 \\
& $4^{\text {th }}$ pic & 0.7296 & 0.7892 & 0.7373 & 1 \\
\hline
\end{tabular}

between volunteer third picture with other pictures in third column of the table. Yellow column represents correlation RV between volunteer fourth picture with other pictures in second column of the table. The black line represents total of average correlation. The degrees located at Y-axis represent value result.

As shown from Table 11 and Fig. 17 below, Correlation average for $10^{\text {th }}$ volunteer Face images shows RV of 0.9 for each image with its corresponding person images, which is a positive result according to the range mentioned above. Blue column represents correlation RV between volunteer first picture with other pictures in first column of the table. Brown column represents correlation RV between volunteer second picture with other pictures in second column of the table. Gray column represents correlation RV between volunteer third picture with other pictures in third column of the table. Yellow column represents correlation RV between volunteer 
fourth picture with other pictures in second column of the table. The black line represents total of average correlation. The degrees located at Y-axis represent value result.

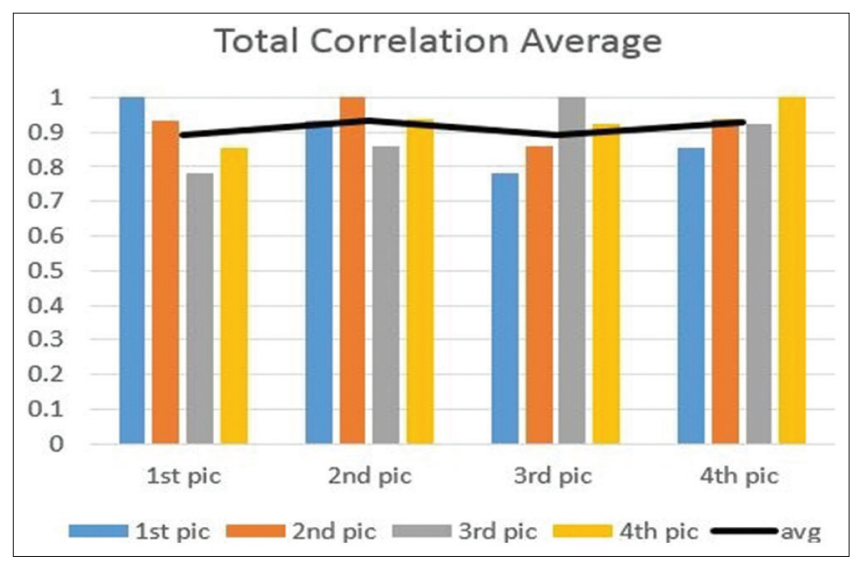

Fig. 17. Average CORRELATION value for $10^{\text {th }}$ volunteer FACE images.

\section{TABLE 11 : Correlation value for $10^{\text {th }}$ volunteer FACE images}

\begin{tabular}{lccccc}
\hline & & $\mathbf{1}^{\text {st }} \mathbf{p i c}$ & $\mathbf{2}^{\text {nd }} \mathbf{p i c}$ & $\mathbf{3}^{\text {rd }} \mathbf{p i c}$ & $\mathbf{4}^{\text {th }}$ pic \\
\hline $10^{\text {th }}$ Volunteer & $1^{\text {st }}$ pic & 1 & 0.9344 & 0.7794 & 0.8566 \\
FACE image & $2^{\text {nd }}$ pic & 0.9344 & 1 & 0.8583 & 0.9354 \\
& $3^{\text {rd }}$ pic & 0.7794 & 0.8583 & 1 & 0.9224 \\
& $4^{\text {th }}$ pic & 0.8566 & 0.9354 & 0.9224 & 1 \\
\hline
\end{tabular}

TABLE 12: Correlation value result for all volunteers

\begin{tabular}{lcccccccccc}
\hline Volunteers & v1 & v2 & v3 & v4 & v5 & v6 & v7 & v8 & v9 & v10 \\
\hline $\begin{array}{l}\text { Correlation } \\
\text { Result }\end{array}$ & 0.9 & 0.9 & 1 & 0.9 & 0.9 & 1 & 0.8 & 0.9 & 0.9 & 0.9 \\
\hline
\end{tabular}

TABLE 13: Comparison some related works with our proposed algorithm

\begin{tabular}{lllc}
\hline No & Authors & Techniques & \% Accuracy \\
\hline 1 & $\begin{array}{l}\text { Swarup Kumar } \\
\text { Dandpat and }\end{array}$ & PCA and 2DPCA & 90.5 \\
& $\begin{array}{l}\text { SukadevMeher [28] } \\
2\end{array}$ & Pallavi D. Wadakar and & DWT \\
& MeghaWankhade [27] & & \\
3 & Chun-Hou Zheng, & ERSC_LR algorithm & 90 \\
& Yi-Fu Hou, and Jun & SVM (linear) & 97.97 \\
& Zhang [18] & NN & 90.37 \\
& & FDDL & 97.01 \\
4 & Omed Hassan Ahmed, & SVD + DWT & 97.72 \\
& Joan Lu, Qiang Xu, & 100 \\
& and Muzhir Shaban & & \\
Al-Ani [25] & Proposed Method & DWT and Median & 91 \\
& & Filter & \\
\hline
\end{tabular}

Table 12 explains total accuracy rate for all 10 volunteers. For the (volunteer 1, volunteer 2, volunteer 4, volunteer 8 , volunteer 9 , and volunteer 10 ). the accuracy is $90 \%$. In addition, the accuracy is $100 \%$ for volunteer 3 , volunteer 6 , and for volunteer 7 is $80 \%$ ).

Total Accuracy rate $=$ total result with error $/ 10=9.1 / 10=$ $(0.91 * 100)=91 \%$

Table 13 demonstrates the comparison works from other researchers and our proposed system. Kumar et al. apply (PCA and 2DPCA) techniques the accuracy is $90.5 \%$ [28]. Wadakar et al. apply DWT technique the accuracy is $90 \%$ [27]. Zheng et al. use (ERSC_LR algorithm, SVM (linear), NN, FDDL, SVM (RBF)) techniques the accuracy is $97.97,94.93,90.28,90.01$, and $97.72 \%$, respectively [18]. Ahmed. et al. apply (SVD and DWT) the accuracy is $1000 \%$ [25]. Finally, (DW'T and Median Filter) techniques apply in our proposed system the accuracy is $91 \%$.

\section{CONCLUSION}

Biometry is an effective method for identification and differentiation among humans, it has done either on physiological properties such as iris, face, finger.... or on behavior properties such as voice, signature.... The obtained identification results for face biometrics are $91 \%$ of accuracy (based on Table 12 average value) by applying DWT and Median filter approach. To compare our accuracy result with other mentioned results a good accuracy score has been achieved. Moreover, all images are captured within the same environment and specification without additional change all images have the same angle and background.

\section{REFERENCES}

[1] R. Malathi and R. R. R. Jeberson. "An integrated approach of physical biometric authentication system" Procedia Computer Science, vol. 85, pp. 820-826, 2016.

[2] F. Battaglia, G. lannizzotto and L. Lo Bello. "A person authentication system based on RFID tags and a cascade of face recognition algorithms". IEEE Transactions on Circuits and Systems for Video Technology, vol. 27, no. 8, pp. 1676-1690, 2017.

[3] A. James, I. Fedorova, T. Ibrayev and D. Kudithipudi. "HTM spatial pooler with memristor crossbar circuits for sparse biometric recognition". IEEE Transactions on Biomedical Circuits and Systems, vol. 11, no. 3, pp. 640-651, 2017.

[4] L. Best-Rowden and A. Jain. "Longitudinal study of automatic face recognition". IEEE Transactions on Pattern Analysis and Machine Intelligence, vol. 40, no. 1, pp. 148-162, 2018.

[5] L. Zhang, P. Dou and I. Kakadiaris. "Patch-based face recognition using a hierarchical multi-label matcher". Image and Vision 
Computing, vol. 73, pp. 28-39, 2018.

[6] A. Punnappurath, A. Rajagopalan, S. Taheri, R. Chellappa and G. Seetharaman. "Face recognition across non-uniform motion blur, illumination, and pose". IEEE Transactions on Image Processing, vol. 24, no. 7, pp. 2067-2082, 2015.

[7] Z. A. Kakarash, D. F. Abd, M. Al-Ani, G. Abubakr and K. M. Omar. "Biometric Iris recognition approach based on filtering techniques". Journal of the University of Garmian, vol. 6, no. 2, p. 34243, 2019.

[8] M. Sharif, S. Bhagavatula, L. Bauer and M. Reiter. "Accessorize to a Crime". Proceedings of the 2016 ACM SIGSAC Conference on Computer and Communications Security-CCS'16, United States, 2016.

[9] A. Aboshosha, K. A. El Dahshan, E. A. Karam and E. A. Ebeid. "Score level fusion for fingerprint, Iris and face biometrics". International Journal of Computer Applications, vol. 111, no. 4, pp. 47-55, 2015.

[10] Y. Xu, Z. Zhang, G. Lu and J. Yang. "Approximately symmetrical face images for image preprocessing in face recognition and sparse representation based classification". Pattern Recognition, vol. 54, pp. 68-82, 2016.

[11] S. Karahan, M. Kilinc Yildirum, K. Kirtac, F. Rende, G. Butun and H. Ekenel. How Image Degradations Affect Deep CNN-based Face Recognition?" 2016 International Conference of the Biometrics Special Interest Group, Darmstadt, Germany, 2016.

[12] R. V. Chawngsangpuii and K. S. Yumnam. "Different approaches to face recognition". International Journal of Engineering Research and Technology, vol. 4, no. 9, pp. 71-75, 2015.

[13] E. Hjelmås. "Biometric Systems: A Face Recognition Approach". Department of Informatics University of Oslo, Oslo, Norway.

[14] O. H. Ahmed, J. Lu, Q. Xu and M. S. Al-Ani. "Face recognition based rank reduction SVD approach". The ISC International Journal of Information Security, vol. 11, no. 3, p. 6, 2019.

[15] P. N. Belhumeur, J. P. Hespanha and D. J. Kriegman. "Eigenfaces vs. Fisherfaces: Recognition using class specific linear projection". IEEE Transactions on Pattern Analysis and Machine Intelligence, vol. 19, p.7, 1997.

[16] S. B. Chen, C. H. Q. Ding and B. Luo. "Extended linear regression for undersampled face recognition". Journal of Visual Communication and Image Representation, vol. 25, no. 7, pp. 1800-1809, 2014.

[17] A. Dehghan, O. Oreifej and M. Shah. "Complex event recognition using con-strained low-rank representation". Image and Vision Computing, vol. 42, pp. 13-21, 2015.

[18] C. H. Zheng, Y. F. Hou and J. Zhang. "Improved sparse representation with low-rank representation for robust face recognition". Neurocomputing, vol. 198, pp. 114-124, 2016.
[19] G. Gao, J. Yang, X. Y. Jing, F. Shen, W. Yang and D. Yue. "Learning robust and discriminative low-rank rep-resentations for face recognition with occlusion". Pattern Recognition, vol. 66, pp. 129143, 2017.

[20] C. Y. Wu and J. J. Ding. "Occluded face recognition using lowrank regression with gen- eralized gradient direction". Pattern Recognition, vol. 80, pp. 256-268, 2018.

[21] P. Jing, Y. Su, Z. Li, J. Liu and L. Nie. "Low-rank regularized tensor discriminant representation for image set classification". Signal Processing, vol. 156, pp. 62-70, 2019.

[22] P. D. Wadkar and M. Wankhade. "Face recognition using discrete wavelet transforms". International Journal of Advanced Engineering Technology, vol. 3, pp. 239-242, 2012.

[23] S. K. Dandapat and S. Meher. "Performance improvement for face recognition using PCA and two-dimensional PCA". IEEE International Conference on Computer Communication and Informatics, vol. 2013, pp. 1-5, 2013.

[24] R. Chellappa, C. L. Wilson, and S. Sirohey. "Human and machine recognition of faces: A survey". Proceedings of the IEEE, vol. 83, no. 5, pp. 405-741, 1995.

[25] A. S. Tolba, A. H. El-Baz and A. A. El-Harby. "Face recognition: A literature review". World Academy of Science, Engineering and Technology, vol. 2, pp. 7-21, 2008.

[26] Z. A. Kakarash, S. H. T. Karim and Mohammadi, M. "Fall detection using neural network based on internet of things streaming data". UHD Journal of Science and Technology, vol. 4, no. 2, pp. 91-98, 2020.

[27] D. Leonidas. "Emerging Trends in Image Processing, Computer Vision and Pattern Recognition. Elsevier, Huntsville, AL, USA, pp. 183-199, 2015.

[28] L. Cornelius. "Multidimensional Systems: Signal Processing and Modeling Techniques". Elsevier, Los Angeles, USA, 1995.

[29] I. Masi, F. Chang, J. Choi, S. Harel, J. Kim, K. Kim, J. Leksut, S. Rawls, Y. Wu, T. Hassner, W. AbdAlmageed, G. Medioni, L. Morency, P. Natarajan and R. Nevatia. "Learning pose-aware models for pose-invariant face recognition in the wild". IEEE Transactions on Pattern Analysis and Machine Intelligence, vol. 41, no. 2, pp. 1-1, 2018.

[30] G. V. Sagar, S. Y. Barker, K. B. Raja, K. S. Babu and K. R. Venugopal. "Convolution Based Face Recognition Using DWT and Feature Vector Compression". Institute of Electrical and Electronics Engineers Conference Paper, United States, 2015.

[31] C. Kyong, K. W. Bowyer and S. Sarkar. "Comparison and combination of ear and face images in appearance-based biometrics". IEEE Transactions on Pattern Analysis and Machine Intelligence, vol. 25, no. 9, pp. 1160-1165, 2013. 